\title{
Current status of viroid taxonomy
}

\author{
F. Di Serio $\cdot$ R. Flores $\cdot$ J. Th. J. Verhoeven $\cdot$ \\ S.-F. Li $\cdot$ V. Pallás $\cdot$ J. W. Randles • \\ T. Sano $\cdot$ G. Vidalakis $\cdot$ R. A. Owens
}

Received: 4 June 2014/ Accepted: 30 July 2014/Published online: 13 September 2014

(C) Springer-Verlag Wien 2014

\begin{abstract}
Viroids are the smallest autonomous infectious nucleic acids known so far. With a small circular RNA genome of about 250-400 nt, which apparently does not code for any protein, viroids replicate and move systemically in host plants. Since the discovery of the first viroid almost forty-five years ago, many different viroids have been isolated, characterized and, frequently, identified as the causal agents of plant diseases. The first viroid classification scheme was proposed in the early 1990s and adopted by the International Committee on Taxonomy of Viruses (ICTV) a few years later. Here, the current viroid
\end{abstract}

Dr. Owens-Agricultural Research Service, USDA, retired.

The Authors of this manuscript are members of the Viroid Study Group of the International Committee on Taxonomy of Viruses (http://www.ictvonline.org/subcommittee.asp?committee=72).

Electronic supplementary material The online version of this article (doi:10.1007/s00705-014-2200-6) contains supplementary material, which is available to authorized users.

\section{F. Di Serio $(\square)$}

Institute for Sustainable Plant Protection, National Research

Council (C.N.R.), 70126 Bari, Italy

e-mail: f.diserio@ba.ivv.cnr.it; francesco.diserio@cnr.it

\section{R. Flores · V. Pallás}

Instituto de Biología Molecular y Celular de Plantas

(UPV-CSIC), Consejo Superior de Investigaciones Científicas, 46022 Valencia, Spain

J. Th. J. Verhoeven

National Plant Protection Organization, National Reference

Centre, P.O. Box 9102, 6700 HC Wageningen, The Netherlands

\section{S.-F. Li}

State Key Laboratory of Biology of Plant Diseases and Insect Pest, Institute of Plant Protection, Chinese Academy of Agricultural Sciences, Beijing 100193, China taxonomy scheme and the criteria for viroid species demarcation are discussed, highlighting the main taxonomic questions currently under consideration by the ICTV Viroid Study Group. The impact of correct taxonomic annotation of viroid sequence variants is also addressed, taking into consideration the increasing application of nextgeneration sequencing and bioinformatics for known and previously unrecognized viroids.

\section{Introduction}

Viroids are small, circular, single-stranded RNAs that replicate autonomously when inoculated into certain higher plants. The first viroid, potato spindle tuber viroid (PSTVd), was discovered in the late 1960s-early 1970s [8], becoming a model for studying replication, pathogenicity and movement (for a review, see [50]) of many other similar infectious agents discovered in subsequent years.

\section{J. W. Randles}

School of Agriculture, Food and Wine, University of Adelaide,

Waite Campus, Adelaide, SA 5064, Australia

T. Sano

Faculty of Agriculture and Life Science, Hirosaki University,

Hirosaki, Japan

G. Vidalakis

Department of Plant Pathology and Microbiology, University of California, Riverside, CA 92521, USA

\section{R. A. Owens}

Molecular Plant Pathology Laboratory, US Department of Agriculture, Agricultural Research Service, Beltsville, MD 20705, USA 
Unlike conventional viruses, there is no evidence that viroids encode any protein, and therefore, their genomes are not protected by a protein capsid. Also in contrast to viruses, which parasitize primarily the translation machinery of their hosts, viroids parasitize host transcription by subverting the template specificity of either the nuclear RNA polymerase II (family Pospiviroidae) or one of the RNA polymerases found in plastids (family Avsunviroidae) to accept RNA templates (for reviews, see [10, 19, 62]. Additionally, the specificity of DNA ligase 1 is subverted to act upon RNA substrates by viroids in the family Pospiviroidae [49].

Presently known viroids vary in length from 246 to 401 nt (excluding those with terminal sequence repeats) and display extensive internal base pairing. Many viroids assume a rod-like or quasi-rod-like conformation in vitro, but at least three viroids adopt clearly branched conformations (Fig. 1). Elements of tertiary structure, such as kissing-loop interactions, may also be present (Fig. 1, lower panel; for a review, see [23]. Certain plant-viral satellite RNAs exhibit many of the same structural features, but, unlike viroids, their replication requires the presence of a specific helper virus (for a review, see [53]). The RNAs of human hepatitis delta virus display common structural (rod-like secondary structure) and functional (presence of ribozymes) characteristics with some viroids, but in contrast, the antigenomic strand codes for a protein [24]

Several viroids, including hop stunt viroid (HpSVd, hereafter referred to as HSVd because this latter acronym is more frequent in the literature) and PSTVd have wide host ranges among the angiosperms, but others, particularly members of the family Avsunviroidae, have narrow host ranges. Viroids spread mainly by vegetative propagation of infected plants or by mechanical transmission by pruning tools. In plants propagated via seeds, transmission through seed or pollen must also be taken into consideration. Some viroids are pathogenic and have significant agricultural
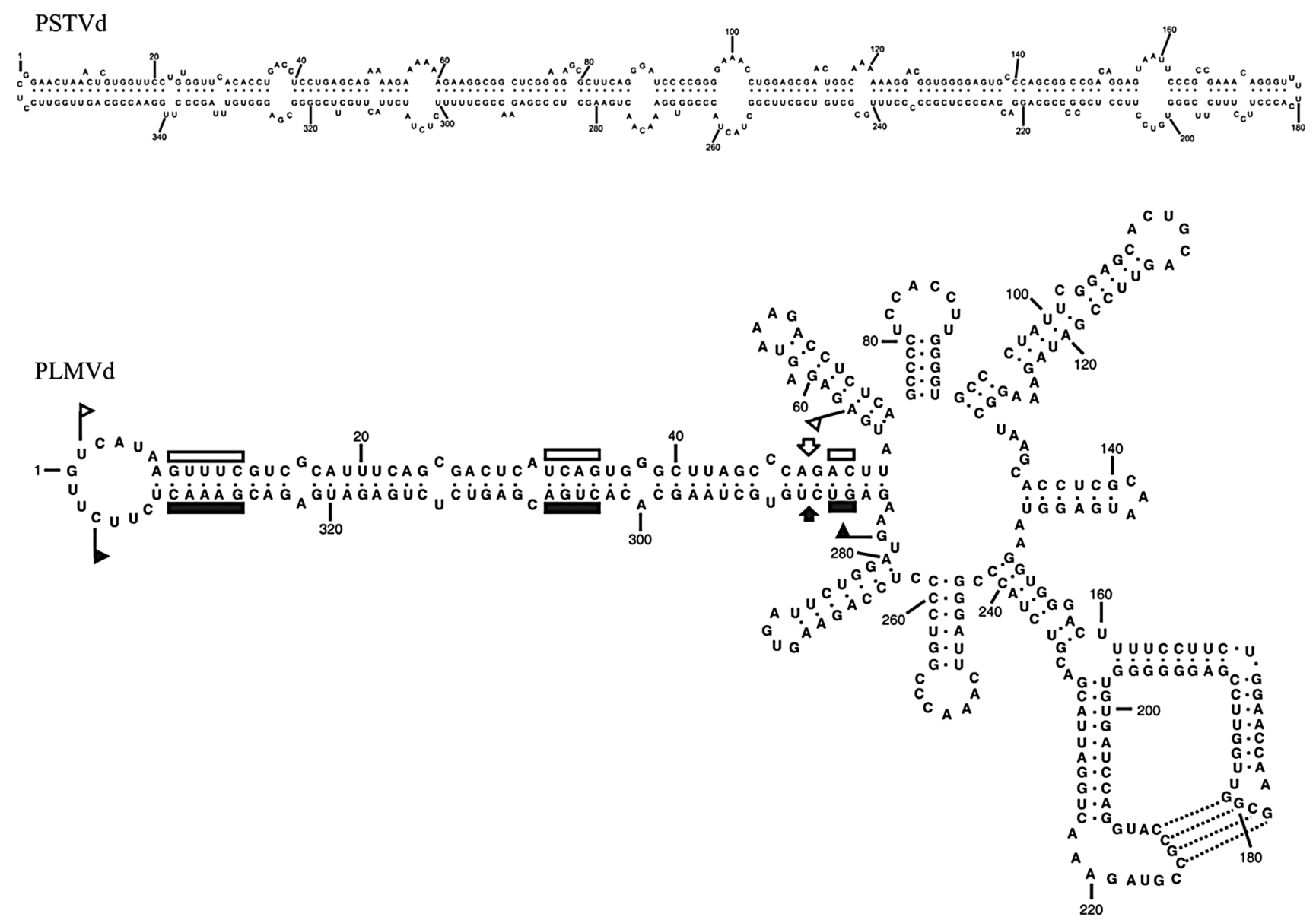

Fig. 1 Primary and secondary structures of PSTVd (above) and PLMVd (below). Plus and minus self-cleavage domains in PLMVd genomic and anti-genomic RNAs are delimited by flags, the eleven conserved residues present in most natural hammerhead structures are indicated by bars, and the self-cleavage sites by arrows. Solid and open symbols refer to plus and minus polarities, respectively. A kissing-loop interaction between positions $178-181$ and 211-214 [3] is shown. (Adapted with modifications from Gross et al. [29] and Hernández and Flores [33]) 
implications, while others replicate without eliciting symptoms in susceptible plants of different species (for a review, see [16]). As described below, there are important practical reasons for generating a classification scheme for viroids that recognizes the continuum existing between biological entities on the one hand and establishes useful criteria for demarcation of taxa on the other.

\section{Current viroid taxonomy scheme}

Table 1 shows the most recent taxonomy of viroids published in the 9th ICTV Report [51]. This scheme is a direct descendant of the first formal proposal for viroid classification advanced by Elena et al. [13]. Following official adoption, this taxonomic scheme first appeared in the 6th ICTV Report [15], in which several viroid species were listed. Higher taxa (genera and families) for viroid classification were formally proposed by Flores et al. [21] and adopted in the 7th ICTV Report [22].

As shown in Figure 2, the rod-like secondary structures of lowest free energy proposed for most members in the

Table 1 Current taxonomy of viroids

\begin{tabular}{|c|c|}
\hline \multicolumn{2}{|l|}{ Family Avsunviroidae } \\
\hline Genus Avsunviroid & 1 species - Avocado sunblotch viroid \\
\hline Genus Pelamoviroid & $\begin{array}{l}2 \text { species - Chrysanthemum chlorotic mottle } \\
\text { viroid, Peach latent mosaic viroid }\end{array}$ \\
\hline Genus Elaviroid & 1 species - Eggplant latent viroid $^{\mathrm{a}}$ \\
\hline \multicolumn{2}{|l|}{ Family Pospiviroidae } \\
\hline Genus Pospiviroid & $\begin{array}{l}10 \text { species - Chrysanthemum stunt viroid, } \\
\text { Citrus exocortis viroid, Columnea latent } \\
\text { viroid, Iresine viroid } 1, \text { Mexican papita } \\
\text { viroid }^{\mathrm{b}}, \text { Pepper chat fruit viroid }{ }^{\mathrm{a}} \text {, Potato } \\
\text { spindle tuber viroid, Tomato apical stunt } \\
\text { viroid, Tomato chlorotic dwarf viroid, } \\
\text { Tomato planta macho viroid }\end{array}$ \\
\hline Genus Hostuviroid $^{\mathrm{c}}$ & 1 species - Hop stunt viroid \\
\hline Genus Cocadviroid & $\begin{array}{l}4 \text { species - Citrus bark cracking viroid, } \\
\text { Coconut cadang-cadang viroid, Coconut } \\
\text { tinangaja viroid, Hop latent viroid }\end{array}$ \\
\hline Genus Apscaviroid & $\begin{array}{l}10 \text { species - Apple dimple fruit viroid, Apple } \\
\text { scar skin viroid, Australian grapevine } \\
\text { viroid, Citrus bent leaf viroid, Citrus } \\
\text { dwarfing viroid, Citrus viroid } V^{\mathrm{a}} \text {, Citrus } \\
\text { viroid VI }{ }^{\mathrm{a}} \text {, Grapevine yellow speckle viroid } \\
1, \text { Grapevine yellow speckle viroid } 2, \text { Pear } \\
\text { blister canker viroid }\end{array}$ \\
\hline Genus Coleviroid & $\begin{array}{l}3 \text { species - Coleus blumei viroid 1, Coleus } \\
\text { blumei viroid 2, Coleus blumei viroid } 3\end{array}$ \\
\hline \multicolumn{2}{|c|}{ a Species recognized after 1998} \\
\hline \multicolumn{2}{|c|}{$\begin{array}{l}\text { b Species under revision; proposals submitted to ICTV for official } \\
\text { approval }\end{array}$} \\
\hline
\end{tabular}

family Pospiviroidae can be divided into five structural domains, namely, C (central), $\mathrm{P}$ (pathogenic), V (variable), and TL and TR (terminal left and right) [41]. The $\mathrm{C}$ domain contains a central conserved region (CCR) formed by two sets of conserved nucleotides located in the upper and lower strands, with those of the upper strand being flanked by an imperfect inverted repeat. A functional role has been proposed for some of these domains in certain members of this family: for example, nucleotide changes in the $\mathrm{P}$ domain have been correlated with strain virulence in citrus exocortis viroid (CEVd) and PSTVd [41, 54, 55]. The upper CCR strand may assume an alternative conformation (hairpin I) with major role(s) in replication [25], and the TL domain may contain either a terminal conserved region (TCR) or a terminal conserved hairpin (TCH). Because the TCR and TCH occupy the same relative position in several members of the family, they are assumed to play some as yet unknown functional role(s). Other motifs, including a so-called RY motif and a polypurine tract are conserved in members of several species in the family Pospiviroidae $[28,41]$.

In contrast to the Pospiviroidae, members of the family Avsunviroidae may assume a branched conformation and are characterized by the presence of hammerhead ribozymes (Fig. 3) in both polarity strands, which allow selfcleavage of the oligomeric RNAs intermediates generated through a rolling-circle replication mechanism. Due to their obvious biological implications, CCR and hammerhead ribozymes have long been considered of taxonomic relevance $[13,21]$. In fact, nowadays, two characteristics the presence/absence of either a CCR or hammerhead ribozymes - divide viroids into two families. Within the Pospiviroidae (the members of which replicate in the nucleus) the type of CCR and the presence or absence of the two other conserved regions (TCR and TCH, see Fig. 2) are used to distribute the various species among five genera. Members of the Avsunviroidae replicate in plastids and lack a CCR. The presence and type of hammerhead ribozymes together with other characteristics, such as the $\mathrm{G}+\mathrm{C}$ content and solubility in $2 \mathrm{M} \mathrm{LiCl}$, are used to allocate the four recognized species among three different genera.

Over the seven years between the two last ICTV reports on viroid taxonomy $[20,51]$ the number of recognized species increased from 28 to 32, and the names of several viroid species were changed to conform to current ICTV guidelines. Moreover, one new genus (Elaviroid) was added to the family Avsunviroidae.

Because several of the criteria suggested by the ICTV for the definition of viral species (e.g., morphological characteristics, protein features and antigenic properties of members) are not applicable to viroids, a fundamental question arises: why does Section 3.32 of the International 

$T_{L}$
$\mathbf{P}$
C
V
$T_{R}$

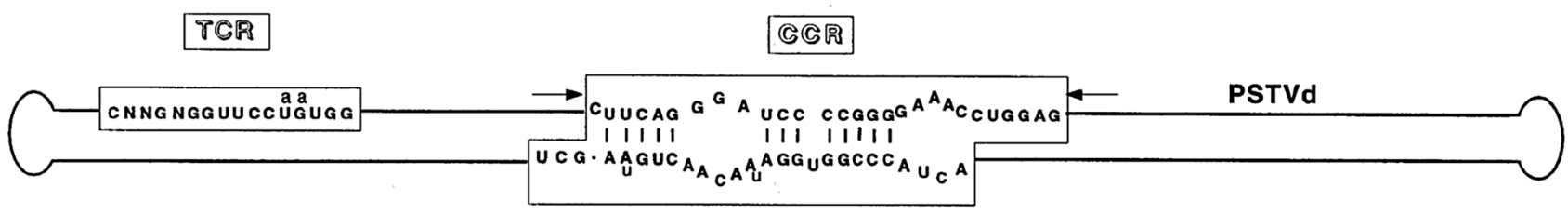

TOM

CGQ

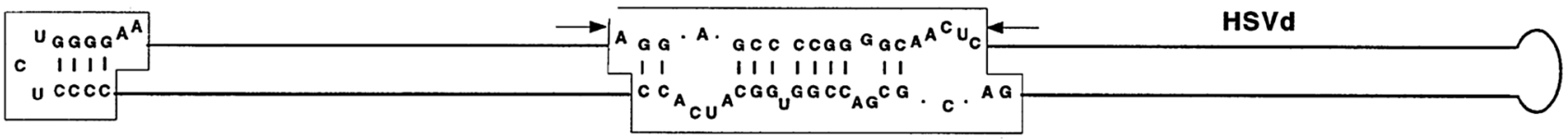

TOH

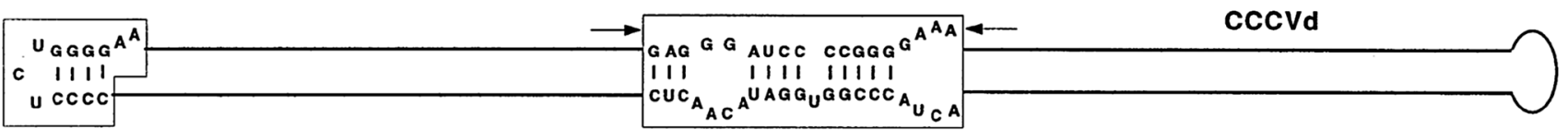

\begin{tabular}{ll} 
TOP & GGR \\
\hline
\end{tabular}
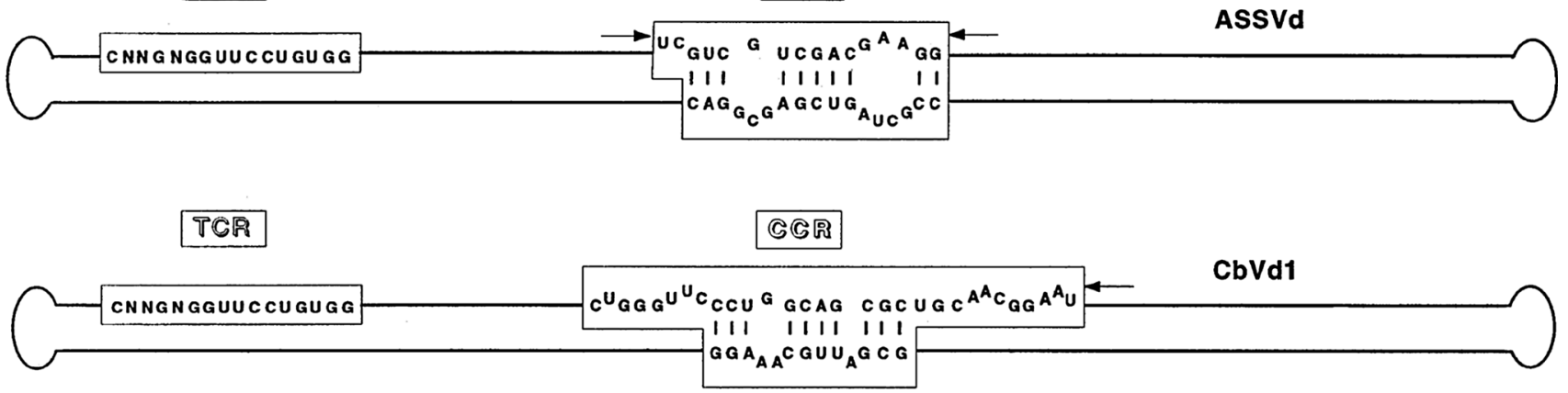

Fig. 2 Schematic structures of viroids in the five genera of the family Pospiviroidae. The type species of each genus is indicated on the right together with the approximate location of the five structural domains shown above. Core sequences of the central conserved region (CCR), terminal conserved region (TCR) and terminal conserved hairpin $(\mathrm{TCH})$ are shown. Arrows indicate flanking

Code of Virus Classification and Nomenclature specifically state that "Rules concerned with the classification of viruses shall also apply to the classification of viroids"? The simplest answer is that like viruses (but unlike other subviral agents such as satellites and prions), viroid genomes replicate autonomously following their introduction into a susceptible host. Moreover, like viruses, viroids propagate in a single host as an ensemble of related variants differing slightly from each other, thus assuming the typical features of quasispecies [2, 6]. Finally, also resembling viruses, mutation and recombination during replication have been identified as the major mechanisms driving evolution and adaptation of viroids to hosts or environmental constraints (reviewed by [16]). sequences, which, together with the core nucleotides of the CCR upper strand, form imperfect inverted repeats. Lower-case fonts indicate substitutions in the CCR and TCR, illustrated here for iresine viroid (IrVd), a member of the genus Pospiviroid. In the genus Coleviroid, the TCR only exists in the two largest members, CbVd-2 and CbVd-3. (Reproduced from [18] with permission from Elsevier.)

\section{Criteria for viroid species demarcation}

Due to their quasispecies nature, viroid populations accumulate in a single infected plant as a spectrum of closely related variants generally showing $>90 \%$ sequence identity, with one or a limited number of these variants often representing the bulk of the population. In formulating the taxonomy of viroids, Flores et al. [21] arbitrarily selected a value of $90 \%$ sequence identity over their entire genome as the sole criterion distinguishing individual viroid species from sequence variants. Biological properties were not explicitly considered. With the exception of certain members of the genus Pospiviroid (vernacular name, pospiviroids), however, members of individual species within the 


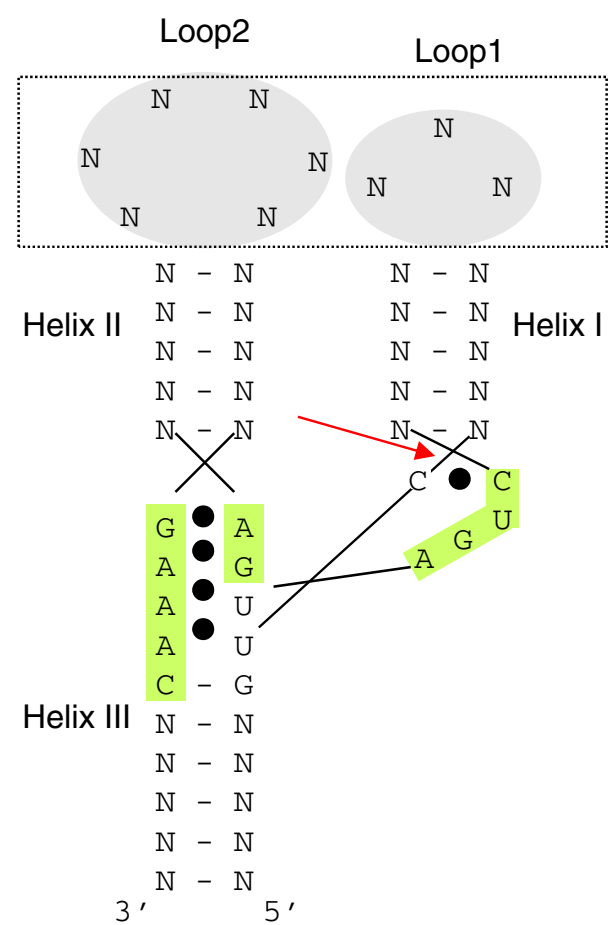

Fig. 3 Schematic representation of the structure of a hammerhead ribozyme. The arrowhead marks the self-cleavage site, and the tertiary interaction between loops 1 and 2 (in grey), which facilitates the catalytic activity of the ribozyme at the low magnesium concentration existing in vivo, is indicated by a dotted rectangle. Continuous lines and dots between nucleotides denote canonical and non-canonical base pairs, respectively. Sequences strictly or highly conserved in natural hammerhead structures are shown within green boxes. N, any nucleotide forming stable helices I, II and III. (Adapted with modifications from [17])

different genera also differ in their biological properties (see next section).

Presently, two criteria - an arbitrary level of less than $90 \%$ sequence identity over the entire genomes and distinct biological properties, particularly host range and symptoms - are used to create new viroid species [51]. In accordance with ICTV guidelines, this second criterion is now mandatory. If host range is restricted to a single botanical species not expressing symptoms, other distinct biological properties should be described to justify creation of a new species; e.g., seed transmission, movement and distribution within the host, or differential fitness in competition assays.

Until recently, the ICTV defined a virus species as "a polythetic class of viruses that constitutes a replicating lineage and occupies a particular ecological niche." The adjective "polythetic" means that the viruses (or viroids) do not need to exhibit a common core set of biological, genetic or structural properties, but rather that their properties overlap to varying degrees. As discussed below, this definition provides a useful framework in which to consider the classification of columnea latent viroid $(\mathrm{CLVd}$, [31] and dahlia latent viroid, DLVd, [63]), two viroids whose CCRs most closely resemble that of HSVd but also, like PSTVd, contain a TCR (see below).

The current ICTV species definition states that: "A species is the lowest taxonomic level in the hierarchy approved by the ICTV. A species is a monophyletic group of viruses whose properties can be distinguished from those of other species by multiple criteria." These criteria shall be established by the appropriate Study Group and may include natural and experimental host range, cell and tissue tropism, pathogenicity, vector specificity, antigenicity, and the degree of relatedness of their genomes or genes. In the case of viroids, although the 32 recognized species and eight genera were established using either the arbitrarilyselected value of $<90 \%$ sequence identity [21] or the previous ICTV definition of virus (and viroid) species, they essentially meet the new requirement for monophyletic origin (Fig. 4; see also [12, 13, 51]). Therefore, the new criteria adopted for defining virus species can be certainly applied to viroids, thus preserving Section 3.32 of the International Code of Virus Classification and Nomenclature. As shown in Figure 4, a monophyletic origin of viroids can be assumed at the genus and family levels, as proposed before [12]. Moreover, the apparently monophyletic origin of viroids and several groups of ribozyme-containing satellite RNAs [13] is an important part of the argument for their possible origin in the "RNA world" [9], but the choice of appropriate phylogenetic approaches for these analyses are a matter of some controversy (e.g., [12, 39]).

Regarding the multiple criteria to be considered for species discrimination, the Viroid Study Group still considers mandatory the need to identify differential biological traits in order to create a new viroid species. This need is particularly relevant to avoid excessive proliferation of new viroid species due to recombination events (see below). Table 2 shows that, excluding the pairs formed by Mexican papita viroid (MPVd)-tomato planta macho viroid (TPMVd), and PSTVdtomato chlorotic dwarf viroid (TCDVd), currently under review by the ICTV Viroid Study Group (see below), the present viroid classification scheme is compatible with a demarcation criterion of around $85 \%$ identity among members of each species. The Study Group is presently discussing the possibility of lowering the indicative threshold of sequence identity over the entire viroid genome required for species demarcation to $<85 \%$.

\section{Unresolved questions}

Three situations raise problems for viroid taxonomy. The first involves the apparently recombinant nature of some viroids, the second concerns the reliability of the host range data used to differentiate certain species of the genus 
Family Pospiviroidae

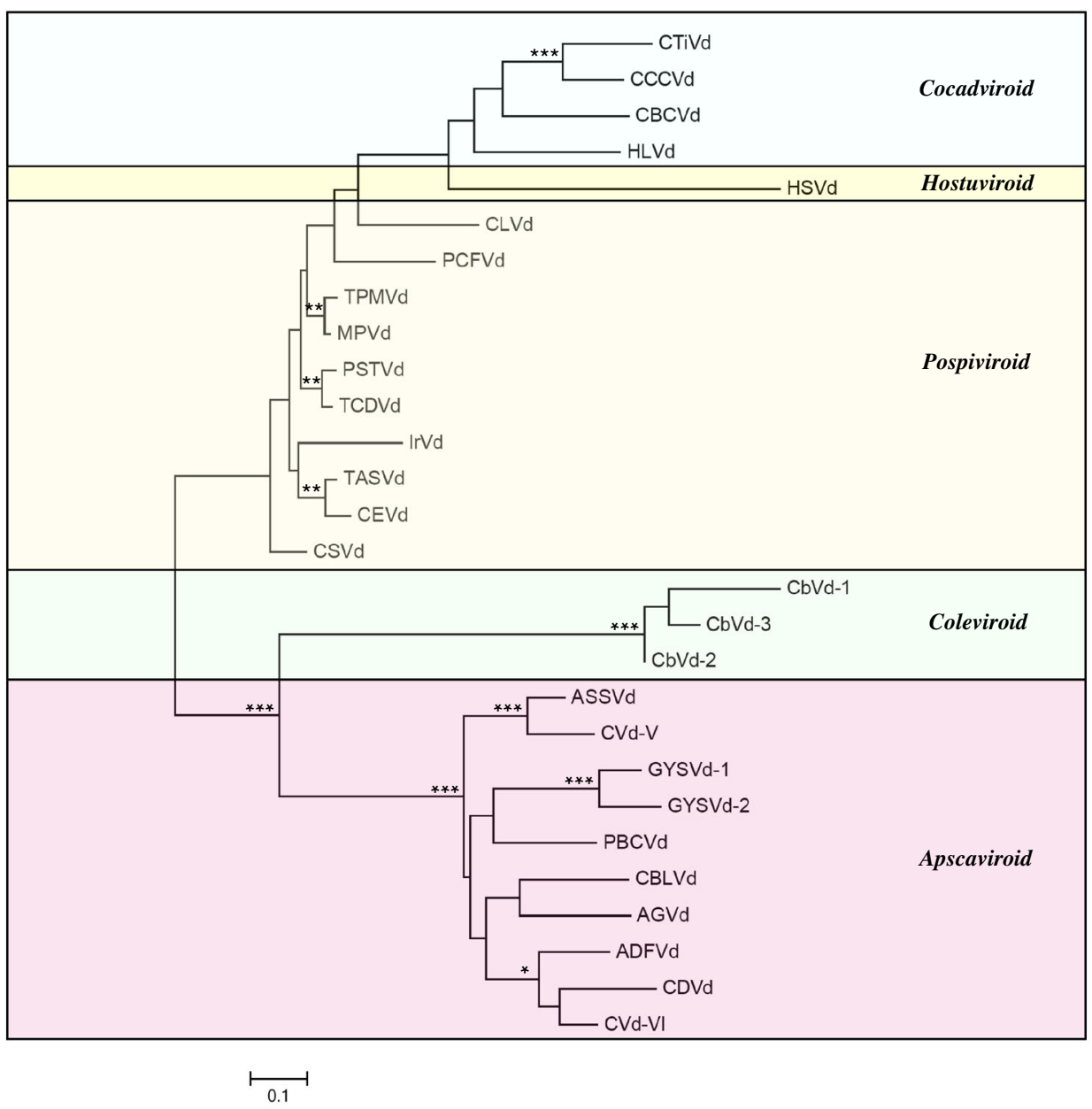

Family Avsunviroidae

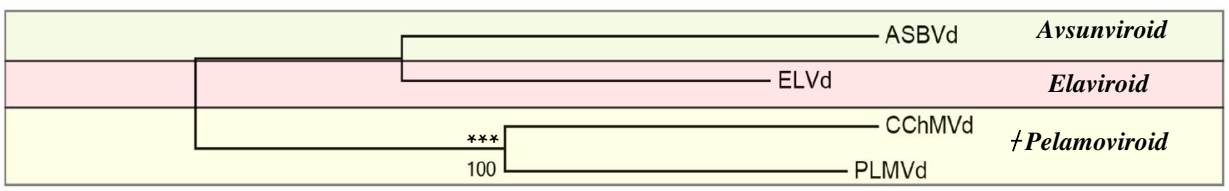

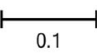

Pospiviroid, and the third refers to the increasingly important role being played by bioinformatics and large-scale RNA sequencing methods in viroid discovery and characterization. We will discuss each of these questions in turn.

\section{Recombinant viroids}

Several years before the first viroid classification scheme was proposed, Keese and Symons [41] had drawn attention to the probable role of RNA recombination in viroid origin/ evolution. Pairwise sequence comparisons strongly suggested that the terminal domains of certain pospiviroids such as tomato apical stunt viroid (TASVd) and TPMVd have undergone recombination in vivo, most likely as the result of discontinuous transcription by the RNA polymerase II involved in viroid replication. On the other hand, the genesis of several viroids, including CLVd [31] and Australian grapevine viroid (AGVd, [52]), was proposed to result from recombination events between viroids 
4Fig. 4 Phylogenetic trees for the viroid families Pospiviroidae and Avsunviroidae. Trees were inferred by the neighbor-joining method using MEGA6 [61] with bootstrap values (1000 replicates). The multiple alignments of reference sequences of each viroid species in the genera of the families Pospiviroidae (upper panel) and Avsunviroidae (lower panel) were performed with ClustalW version 1.6 (multiple alignment gap opening and extension penalties 15 and 1 , respectively, and minor manual adjustments between conserved regions in panel B). Evolutionary distances were estimated according to the model of Jukes and Cantor [40]. Branches marked by ***, ** and * indicate monophyletic groups in more than $95 \%, 85 \%$ and $75 \%$ of the replicates, respectively. Viroids cluster according to their respective taxonomic position within the recognized genera (denoted on the right of each colored box). The acronyms refer to extended names of viroids: apple dimple fruit viroid (ADFVd), apple scar skin viroid (ASSVd), Australian grapevine viroid (AGVd), avocado sunblotch viroid (ASBVd), chrysanthemum chlorotic mottle viroid (CChMVd), citrus bark cracking viroid $(\mathrm{CBCVd})$, citrus bent leaf viroid (CBLVd), citrus dwarfing viroid (CDVd), citrus viroid V $(\mathrm{CVd} \mathrm{V})$, citrus viroid $\mathrm{VI}(\mathrm{CVd}$ $\mathrm{VI})$, coconut cadang-cadang viroid (CCCVd), coconut tinangaja viroid (CTiVd), coleus blumei viroid 1 (CbVd-1), coleus blumei viroid 2 (CbVd-2), coleus blumei viroid 3 (CbVd-3), grapevine yellow speckle viroid 1 (GYSVd-1), grapevine yellow speckle viroid 2 (GYSVd-2), eggplant latent viroid (ELVd), hop latent viroid (HLVd), hop stunt viroid (HSVd), peach latent mosaic viroid (PLMVd), pear blister canker viroid (PBCVd). The acronyms of members of the genus Pospiviroid refer to extended names of viroids according to Table 2

belonging to species of different genera co-infecting the same host. Internal recombination events within the same viroid, generating terminal repeats, have also been reported for some nuclear-replicating viroids, such as coconut cadang-cadang viroid (CCCVd) and CEVd [14, 32, 57].

The apparent prevalence of recombination events in taxonomy is particularly well-exemplified by CLVd [31].
As shown in Table 1, CLVd is currently classified in the genus Pospiviroid in accordance with phylogenetic analysis that places this viroid in the same monophyletic group as PSTVd and other members of this genus (Fig. 4, see also [51]). Moreover, CLVd also has the TCR present in all pospi- and apscaviroids (vernacular name for members of the genus Apscaviroid), as well as in the two largest coleviroids (vernacular name for members of the genus $\mathrm{Co}$ leviroid), but absent from HSVd. From a biological point of view, CLVd also shares with pospiviroids the ability to infect solanaceous hosts, inducing symptoms similar to those caused by most members of this genus. In contrast, the main argument supporting an alternative classification is that CLVd and HSVd share a common CCR (the main structural feature adopted for genus demarcation within the family Pospiviroidae).

In setting up their classification scheme for viroids, Flores et al. [21] clearly recognized the challenges posed by recombinant viroids like CLVd (and the more recently described DLVd) for viroid taxonomy. The decision not to classify CLVd in the genus Hostuviroid considered both its molecular and biological properties [21]. Over time, we can say that this choice was fortunate and has had important practical consequences. For example, CLVd shares many biological properties with other viroids in the genus Pospiviroid, and assignment of CLVd to this genus resulted in its inclusion in a recent risk assessment of solanaceous pospiviroids for the EU territory. Conducted by the European Food Safety Authority (EFSA), this risk assessment project proposed a series of common management options to contain the spread of these viroids in Europe [11].

Table 2 Pairwise sequence identities among pospiviroids ${ }^{\mathrm{a}}$

\begin{tabular}{|c|c|c|c|c|c|c|c|c|c|c|}
\hline Viroid $^{b}$ & CSVd & CEVd & CLVd & $\mathrm{IrVd}$ & MPVd & PCFVd & PSTVd & TASVd & TCDVd & TPMVd \\
\hline CSVd & - & 0.728 & 0.674 & 0.632 & 0.691 & 0.612 & 0.733 & 0.787 & 0.728 & 0.697 \\
\hline CEVd & & - & 0.657 & 0.643 & 0.728 & 0.532 & 0.716 & 0.817 & 0.722 & 0.719 \\
\hline CLVd & & & - & 0.584 & 0.711 & 0.425 & 0.774 & 0.700 & 0.750 & 0.706 \\
\hline IrVd & & & & - & 0.650 & 0.509 & 0.652 & 0.631 & 0.678 & 0.661 \\
\hline MPVd & & & & & - & 0.690 & 0.836 & 0.783 & 0.814 & 0.928 \\
\hline PCFVd & & & & & & - & 0.598 & 0.644 & 0.641 & 0.690 \\
\hline PSTVd & & & & & & & - & 0.716 & 0.897 & 0.797 \\
\hline TASVd & & & & & & & & - & 0.733 & 0.758 \\
\hline TCDVd & & & & & & & & & - & 0.775 \\
\hline TPMVd & & & & & & & & & & - \\
\hline
\end{tabular}

\footnotetext{
${ }^{a}$ Pairwise identities between GenBank reference genomes (http://www.ncbi.nlm.nih.gov/genomes/GenomesGroup.cgi?taxid=12884\&opt=Vir oid) were calculated using DNASTAR Lasergene software after ClustalW alignment. Viroid pairs sharing sequence identity close to 0.90 , which are specifically discussed in the text, are highlighted in bold. Note that the identity values may vary depending on the alignment parameters adopted and the sequence variants considered

${ }^{\mathrm{b}}$ CSVd, chrysanthemum stunt viroid; CEVd, citrus exocortis viroid; CLVd, columnea latent viroid; IrVd, iresine viroid; MPVd, Mexican papita viroid; PCFVd, pepper chat fruit viroid; PSTVd, potato spindle tuber viroid; TASVd, tomato apical stunt viroid; TCDVd, tomato chlorotic dwarf viroid; TPMVd, tomato planta macho viroid
} 
Similar to the situation with CLVd, the need to formally consider whether DLVd belongs to a novel species [63] offers an opportunity to revisit this decision in the light of new evidence. DLVd, with less than $56 \%$ sequence identity to other viroids, displays characteristic features of members of the family Pospiviroidae, including a predicted rod-like secondary structure of minimum free energy with a CCR identical to that of HSVd (genus Hostuviroid). However, DLVd has the TCR present in members of the genus Pospiviroid, but absent in HSVd, and lacks the TCH present in HSVd. Phylogenetic reconstructions indicate that HSVd and pepper chat fruit viroid (PCFVd) (genus Pospiviroid) are the closest relatives of DLVd [63], but DLVd differs from these viroids in its host range, which is restricted to dahlia so far. Therefore, DLVd fulfils the criteria to belong to a novel species of the family Pospiviroidae. Because DLVd, in contrast to CLVd, does not share biological properties with members of the genus Pospiviroid, we propose to assign it to the genus Hostuviroid based on the most important demarcating criterion for this purpose, i.e., a common CCR with the type member of this genus.

An additional taxonomic case linked to the recombinant nature of viroids is the assignment of Citrus bark cracking viroid (CBCVd), (formerly Citrus viroid IV) to the genus Cocadviroid. In this case, a question was raised about the inclusion of CBCVd within this genus because of the biological similarity (host range) of this viroid to members of the genus Pospiviroid (i.e., CEVd) [56]. However, the structural properties and phylogenetic relationships support the present assignment of $\mathrm{CBCVd}$ to the genus Cocadviroid.

The best example of recombination playing a relevant role in the genetic variability and evolution of viroids is illustrated by species in the genus Coleviroid. Three species have been recognized by ICTV in a previous classification scheme based on the arbitrary limit of $90 \%$ sequence identity [21]. Since its first description, it has been clear that coleus blumei viroid $2(\mathrm{CbVd}-2)$ is a viroid chimera composed of the right and left halves of the rodlike secondary structures of coleus blumei viroid 1 (CbVd1) and coleus blumei viroid 3 (CbVd-3), respectively [60]. Even though biological differences between these three viroids have never been demonstrated, they have been maintained as members of separate species. Presently, two other viroids that might be members of the genus Coleviroid are known, but neither has been approved as members of new species: coleus blumei viroid $5(\mathrm{CbVd}-5)$ and coleus blumei viroid 6 (CbVd-6) $[34,35]$. These viroids share less than $90 \%$ sequence identity and can be phylogenetically separated from the previously accepted viroid species (including $\mathrm{CbVd}-1, \mathrm{CbVd}-2$ and $\mathrm{CbVd}-3$ ) [34]. No differential biological properties supporting their classification as members of new species (as required by the most recent ICTV rules) have been identified, however. Interestingly, CbVd-6 is a chimeric molecule isolated from naturally infected Coleus spp. plants that contains the left half of $\mathrm{CbVd}-3$ joined to the right half of $\mathrm{CbVd}-5$. Autonomous replication and seed transmission of both CbVd-5 and CbVd-6 have recently been demonstrated [38]. The viroid known as coleus blumei viroid A1 (also referred to as coleus blumei viroid 4-1) is a case apart because it derives from a chimeric cDNA construct generated in the laboratory, thus representing a synthetic viroid [60] that will not be included in future viroid classification schemes.

\section{Validity of recognized species of genus Pospiviroid}

As shown in Table 1, nearly all of the ten currently recognized species of this genus were established on the basis of sequence similarity alone. In only three cases (i.e., TCDVd, PCFVd, and MPVd) were biological data explicitly considered. Recent observations have begun to raise questions about the strength of the evidence supporting at least one of these species.

Table 2 compares the pairwise sequence identities among all ten recognized pospiviroids. Note that two viroid pairs (i.e., MPVd/TPMVd and TCDVd/PSTVd) are much more closely related than any others. In proposing that MPVd and TPMVd be recognized as members of separate species, Martinez-Soriano et al. [46] pointed to certain differences in their biological properties, namely, the ability of TPMVd (but not MPVd) to infect Gomphrena globosa and the appearance of flower break in Nicotiana glutinosa inoculated with MPVd or PSTVd (but not with TPMVd). Unfortunately, the lack of experimental details makes it difficult to evaluate the strength of this evidence. Back-inoculations to tomato were carried out to confirm the presence/absence of viroid for assays on G. globosa, but the situation for N. glutinosa inoculated with MPVd or PSTVd is unclear. The number of plants tested was also not specified.

Over the past 10-15 years, a steady stream of reports has documented an increasing incidence of pospiviroid infections affecting crops grown under glass/plastic. In addition to tomato, a variety of ornamental species (particularly solanaceous ornamentals) are infected by different pospiviroids [11, 64, 65]. MPVd was originally isolated from Solanum cardiophyllum growing wild in the Mexican state of Aguascalientes [46], but in 2008, two groups $[42,66]$ detected its presence in samples of cultivated tomato collected elsewhere in that country. To 
critically compare the biological properties of MPVd and TPMVd, Verhoeven et al. [66] carried out two independent series of bioassays involving five different pospiviroids and seven commonly used solanaceous and nonsolanaceous indicator hosts; in addition to TPMVd and MPVd (three isolates), CLVd, TCDVd, and PSTVd (three isolates) were also tested. The results indicated that the ability to infect cucumber or Nicotiana tabacum cv White Burley can be used to distinguish CLVd and TCDVd from the other pospiviroids tested, but assays on five other hosts (including G. globosa and N. glutinosa) failed to differentiate among the other pospiviroids. Because it was not possible to discriminate between MPVd and TPMVd based on their infectivity in G. globosa and $N$. glutinosa, these recent data do not support the biological divergence between these two viroids required by ICTV rules for their recognition as members of separate species [66]. The possibility of including them in a single viroid species in the genus Pospiviroid is currently under consideration by the Viroid Study Group.

As shown in Table 2, the nucleotide sequences of two other pospiviroids (i.e., TCDVd and PSTVd) are also closely related. In their initial characterization of TCDVd, Singh et al. [59] reported that, in contrast to PSTVd, the former was not seed-transmissible; this was the case for both potato (two varieties) and five solanaceous indicator species that could be infected with TCDVd by mechanical inoculation. A later publication from the same group [58] showed that TCDVd is actually transmissible through tomato seed.

Verhoeven et al. [66] reported that a randomlyselected isolate of TCDVd could be distinguished from PSTVd (intermediate strain) by its ability to infect $N$. tabacum cv White Burley following mechanical inoculation. However, repeating these assays using additional isolates of TCDVd and two different cultivars of tobacco (i.e., White Burley and Petit Havana SR1) revealed that systemic infection of tobacco requires an A (present in most but not all isolates of TCDVd) rather than an $\mathrm{U}$ (present in most but not all PSTVd isolates) in the loop E motif (position 257 in the PSTVd accession M16826) (Verhoeven, unpublished results). Consequently, the ability to infect tobacco is not sufficient to differentiate among all isolates of these two viroids. Comparatively little is currently known about the sequence motifs that control viroid's ability to replicate and move systemically in different hosts (e.g., [69]); thus, until this situation improves, caution is necessary in evaluating evidence for differences in the biological properties of viroids. At present, the strongest evidence of biological differences between PSTVd and TCDVd is the failure of the former to cross-protect tomato against challenge inoculation with the latter [59].

\section{The impact of taxonomy in viroid detection through large-scale RNA sequence analysis}

Like plant viruses, both nuclear- and chloroplast- replicating viroids are associated with viroid-derived small RNAs (vd-sRNAs) of 21-24 nt that accumulate in infected plant tissues. These vd-sRNAs are structurally similar to host microRNAs and small interfering RNAs [37, 43, 44], the key effectors of RNA silencing [4], thus supporting the involvement of this RNA-based regulatory network in plant-viroid interactions (reviewed by [27, 30, 47]). Besides contributing to further dissecting the molecular events underlying viroid infection, these findings also have major implications for disease diagnosis.

Since the first characterization of vd-sRNAs by nextgeneration sequencing (NGS), these small RNAs were found to map along the whole length of both genomic and antigenomic viroid strands [7, 45, 48]. Therefore, besides being molecular markers of ongoing viroid infections, overlapping vd-sRNAs can be assembled in silico into viroid contigs to subsequently search for similar sequences in databanks (as previously shown for plant viruses). Several viroids have been detected by this approach in the last few years [1, 5, 26, 36, 48, 68]. In 2012, an algorithm was developed to assemble circular RNA genomes from small RNAs resulting from NGS, thus allowing identification of viroid-like RNAs independent of any homology with previously reported sequences [67]. It can be anticipated that more and more viroids and viroid-like RNAs will be detected using these innovative technologies, which largely rely on in silico analysis and databank queries. In this context, the correct taxonomic annotation of sequences in databanks plays a major role in preventing serious misunderstandings similar to those that have occurred in the past (see below).

Unfortunately, despite lacking typical sequence or structural elements that include those of taxonomic relevance, some sequences have been erroneously annotated in databanks as viroids (see Supplementary File online). They are usually artifacts consisting of cDNA fragments of host RNAs (i.e., ribosomal RNAs) amplified in a nonspecific way using primers derived from viroid sequences, as illustrated by sequences wrongly annotated as citrus exocortis viroid (DQ318790 to DQ318794; AY513268) or citrus exocortis Yucatan viroid (FJ751926-FJ751934; FJ662762). Contigs with high sequence identity to those host-derived sequences (erroneously annotated as viroids) can be subsequently generated using NGS data from plant small RNA (21-24 nt) libraries, thus leading to confusion for other researchers not familiar with viroids.

The ICTV Viroid Study Group has undertaken efforts to address this issue. An active collaboration with the National Center for Biotechnology Information (NCBI) has 
already resulted in the exclusion of more than fifty sequences, identified as wrongly annotated as viroids (including those sequences indicated above), from the NCBI Entrez Viral Genomes databank (http://www.ncbi. nlm.nih.gov/genomes/GenomesHome.cgi?taxid=10239). A great benefit to the scientific community is expected by this action because the latter databank is widely used for identifying contigs sharing sequence identity to viruses and viroids. In addition, more stringent guidelines for depositing viroid sequences in databanks should help to prevent similar problems in the future. In this respect, it may be desirable to support proposed taxonomic assignments by demonstrating the presence of taxonomically relevant regions, i.e., CCR, TCR, TCH or hammerhead structures. Hopefully, these guidelines will become mandatory rules in the future. In the meantime, we strongly recommend that they be followed (see Supplementary File online for guidelines to annotate viroid sequences correctly). Finally, some general precautions should also be adopted when a potential viroid sequence is detected by NGS and bioinformatics tools, including confirmation of the presence in plant tissues of the circular RNA forms by northern-blot hybridization with specific probes and/or RT-PCR amplification, using specific adjacent primers of both polarities (designed from the contig sharing high sequence identity with a previously reported viroid), followed by sequencing of the amplified cDNAs. By supplying these data, new circular RNAs containing regions of taxonomic relevance can be tentatively added to the list of viroids that may eventually represent recognized species following formal ICTV approval (which needs fulfillment of the biological criteria indicated above). One example in this context is a viroid-like RNA recently identified in grapevine [67] that shares relevant structural similarities with PLMVd and CChMVd (genus Pelamoviroid), including the presence of hammerhead ribozymes in both polarity strands and a kissing loop interaction.

\section{Conclusions and recommendations}

The ideas discussed above, which were specifically addressed by the ICTV Viroid Study Group at the International Workshop on Viroids and Satellite RNAs held in Beijing, China (August $23^{\text {rd }}-25^{\text {th }} 2013$ ), extend to viroids the new ICTV definition of virus species. As stated in Section 3.32 of the International Code of Virus Classification and Nomenclature "Rules concerned with the classification of viruses shall also apply to the classification of viroids". In this respect, the Viroid Study Group considers mandatory the demonstration of different biological properties when new viroid species are proposed, in order to avoid controversial issues derived from focusing exclusively on phylogenetic and structural properties. Analysis of biological properties should facilitate demarcation of taxa in the continuum existing between biological entities with less ambiguity than other criteria.

Acknowledgments We want to acknowledge the contribution of Drs. Enza M. Torchetti, Santiago F. Elena and Marcos de la Peña for assistance with the phylogenetic analysis. We are grateful to Dr. Yiming Bao of the National Center for Biotechnology Information (NCBI) for his support and advice.

\section{References}

1. Al Rwahnih M, Daubert S, Golino D, Rowhani A (2009) Deep sequencing analysis of RNAs from a grapevine showing Syrah decline symptoms reveals a multiple virus infection that includes a novel virus. Virology 387:395-401

2. Biebricher CK, Eigen M (2006) What is a quasispecies? Curr Top Microbiol Immunol 299:1-31

3. Bussière F, Ouellet J, Côté F, Lévesque D, Perreault JP (2000) Mapping in solution shows the peach latent mosaic viroid to possess a new pseudoknot in a complex branched secondary structure. J Virol 74:2647-2654

4. Carthew RW, Sontheimer EJ (2009) Origins and mechanisms of miRNAs and siRNAs. Cell 136:642-655

5. Chiumenti M, Torchetti EM, Di Serio F, Minafra A (2014) Identification and characterization of a viroid resembling Apple dimple fruit viroid in fig (Ficus carica L) by next generation sequencing of small RNAs. Virus Res. doi:10.1016/jvir usres201403026 (in press)

6. Codoñer FM, Daròs JA, Solé RV, Elena SF (2006) The fittest versus the flattest: experimental confirmation of the quasispecies effect with subviral pathogens. PLoS Path 2:1187-1193

7. Di Serio F, Gisel A, Navarro B, Delgado S, Martínez de Alba AE, Donvito G, Flores R (2009) Deep sequencing of the small RNAs derived from two symptomatic variants of a chloroplastic viroid: implications for their genesis and for pathogenesis. PLoS One 4:e7539

8. Diener TO (1971) Potato spindle tuber "virus" IV. A replicating low molecular weight RNA. Virology 45:411-428

9. Diener TO (1989) Circular RNAs: relics of precellular evolution? Proc Natl Acad Sci USA 86:9370-9374

10. Ding B (2009) The biology of viroid-host interactions. Annu Rev Phytopathol 47:105-131

11. EFSA Panel on Plant Health (PLH) (2011) Scientific Opinion on the assessment of the risk of solanaceous pospiviroids for the EU territory and the identification and evaluation of risk management options. EFSA J 9:2330. doi:10.2903/j.efsa.2012.3027 [133 pp]

12. Elena SF, Dopazo J, De la Peña M, Flores R, Diener TO, Moya A (2001) Phylogenetic analysis of viroid and viroid-like satellite RNAs from plants: a reassessment. J Mol Evol 53:155-159

13. Elena SF, Dopazo J, Flores R, Diener TO, Moya A (1991) Phylogeny of viroids and viroid-like satellite RNAs and the viroid-like domain of hepatitis $\delta$ virus RNA. Proc Natl Acad Sci USA 88:5631-5634

14. Fadda Z, Daròs JA, Flores R, Duran-Vila N (2003) Identification in eggplant of a variant of citrus exocortis viroid (CEVd) with a 96 nucleotide duplication in the right terminal region of the rodlike secondary structure. Virus Res 97:145-149

15. Flores R (1995) Subviral agents: viroids In: Murphy FA, Fauquet CM, Bishop DHL, Ghabrial SA, Jarvis AW, Martelli GP, Mayo MA, Summers MD (eds) Virus taxonomy, 6th report of the 
International Committee on Taxonomy of Viruses, Archives of Virology, Supplement 10, Springer, Vienna AT pp 495-497

16. Flores R, Di Serio F, Navarro B, Duran-Vila N, Owens RA (2011) Viroids and viroid diseases of plants. In: Hurst CJ (ed) Studies in viral ecology 1 microbial and botanical host systems. Wiley \& Sons Inc Hoboken, New Jersey USA, pp 307-342

17. Flores R, Daròs JA, Hernández C, Di Serio F (2011b) Viroids. In: Encyclopedia of life sciences. Wiley, Chichester, [http://www. els.net/]. doi:10.1002/9780470015902.a0000434.pub3

18. Flores R, Di Serio F, Hernández C (1997) Viroids: The noncoding genomes. Semin Virol 8:65-73

19. Flores R, Hernández C, Martínez de Alba AE, Daròs JA, Di Serio F (2005) Viroids and viroid-host interactions. Annu Rev Phytopathol 43:117-139

20. Flores R, Randles JW, Owens RA, Bar-Joseph M, Diener TO (2005b) Viroids In: Fauquet CM, Mayo MA, Maniloff J, Desselberger U, Ball AL (eds) Virus taxonomy classification and nomenclature VIIII report of the International Committee on Taxonomy of Viruses, Elsevier/Academic Press, London UK, pp 1145-1159

21. Flores R, Randles JW, Bar-Joseph M, Diener TO (1998) A proposed scheme for viroid classification and nomenclature. Arch Virol 143:623-629

22. Flores R, Randles JW, Owens RA, Bar-Joseph M, Diener TO (2000) Subviral agents: Viroids In: van Regenmortel MHV, Fauquet CM, Bishop DHL, Carstens EB, Estes MK, Lemon SM, Mc Geoch DJ, Maniloff J, Mayo MA, Pringle CR, Wickner RB (eds) Virus taxonomy, seventh report of the International Committee on Taxonomy of Viruses, Academic Press, San Diego USA, pp 1009-1024

23. Flores R, Serra P, Minoia S, Di Serio F, Navarro B (2012) Viroids: from genotype to phenotype just relying on RNA sequence and structural motifs. Frontiers Microbiol 3:1-13

24. Flores R, Ruiz-Ruiz S, Serra P (2012) Viroids and hepatitis delta virus. Semin Liver Dis 32:201-210

25. Gas ME, Hernández C, Flores R, Daròs JA (2007) Processing of nuclear viroids in vivo: An interplay between RNA conformations. PLoS Pathog 3:1813-1826

26. Giampetruzzi A, Roumi V, Roberto R, Malossini U, Yoshikawa N, La Notte P, Terlizzi F, Credi R, Saldarelli P (2012) A new grapevine virus discovered by deep sequencing of virus- and viroid-derived small RNAs in cv Pinot gris. Virus Res 163:262-268

27. Gomez G, Martinez G, Pallás V (2009) Interplay between viroidinduced pathogenesis and RNA silencing pathways. Trends Plant Sci 14:264-269

28. Gozmanova M, Denti MA, Minkov IN, Tsagris M, Tabler M (2003) Characterization of the RNA motif responsible for the specific interaction of potato spindle tuber viroid RNA (PSTVd) and the tomato protein Virp1. Nucleic Acids Res 31:5534-5543

29. Gross HJ, Domdey H, Lossow C, Jank P, Raba M, Alberty H, Sänger HL (1978) Nucleotide sequence and secondary structure of potato spindle tuber viroid. Nature 273:203-208

30. Hammann C, Steger G (2012) Viroid-specific small RNA in plant disease. RNA Biol 9:809-819

31. Hammond R, Smith DR, Diener TO (1989) Nucleotide sequence and proposed secondary structure of Columnea latent viroid: a natural mosaic of viroid sequences. Nucleic Acids Res 17:10083-10094

32. Haseloff J, Mohamed NA, Symons RH (1982) Viroid RNAs of cadang-cadang disease of coconuts. Nature 299:316-321

33. Hernández C, Flores R (1992) Plus and minus RNAs of peach latent mosaic viroid self-cleave in vitro via hammerhead structures. Proc Natl Acad Sci USA 89:3711-3715

34. Hou WY, Sano T, Li SF, Li F, Li L, Wu ZJ (2009) Identification and characterization of a new Coleviroid (CbVd-5). Arch Virol $154: 315-320$
35. Hou WY, Li SF, Wu ZJ, Jiang DM, Sano T (2009) Coleus blumei viroid 6: a new tentative member of the genus Coleviroid derived from natural genome shuffling. Arch Virol 154:993-997

36. Ito T, Suzaki K, Nakano M, Sato A (2013) Characterization of a new apscaviroid from American persimmon. Arch Virol 158:2629-2631

37. Itaya A, Zhong $\mathrm{X}$, Bundschuh R, Qi $\mathrm{Y}$, Wang $\mathrm{Y}$, Takeda R, Harris AR, Molina C, Nelson RS, Ding B (2007) A structured viroid RNA is substrate for Dicer-like cleavage to produce biologically active small RNAs but is resistant to RISC-mediated degradation. J Virol 81:2980-2994

38. Jiang D, Gao R, Qin L, Wu Z, Xie L, Hou W, Li S-F (2014) Infectious cDNA clones of four viroids in Coleus blumei and molecular characterization of their progeny. Virus Res 180:97-101

39. Jenkins GM, Woelk CH, Rambaut A, Holmes EC (2000) Testing the extent of sequence similarity among viroids satellite RNAs and the hepatitis delta virus. J Mol Evol 50:98-102

40. Jukes TH, Cantor CR (1969) Evolution of protein molecules. In: Munro HN (ed) Mammalian protein metabolism. Academic Press, New York, pp 21-132

41. Keese P, Symons RH (1985) Domains in viroids: evidence of intermolecular RNA rearrangements and their contribution to viroid evolution. Proc Natl Acad Sci USA 82:4582-4586

42. Ling K-S, Zhang W (2009) First report of a natural infection by Mexican papita viroid and Tomato chlorotic dwarf viroid on greenhouse tomatoes in Mexico. Plant Dis 93:1216

43. Machida S, Yamahata N, Watanuki H, Owens RA, Sano T (2007) Successive accumulation of two size classes of viroid-specific small RNA in potato spindle tuber viroid-infected tomato plants. J Gen Virol 88:3452-3457

44. Martín R, Arenas C, Daròs JA, Covarrubias A, Reyes JL, Chua NH (2007) Characterization of small RNAs derived from citrus exocortis viroid (CEVd) in infected tomato plants. Virology 367:135-146

45. Martinez G, Donaire L, Llave C, Pallas V, Gomez G (2010) Highthroughput sequencing of Hop stunt viroid-derived small RNAs from cucumber leaves and phloem. Mol Plant Pathol 11:347-359

46. Martínez-Soriano JP, Galindo-Alonso J, Maroon CJ, Yucel I, Smith DR, Diener TO (1996) Mexican papita viroid: putative ancestor of crop viroids. Proc Natl Acad Sci USA 93:9397-9401

47. Navarro B, Gisel A, Rodio ME, Delgado S, Flores R, Di Serio F (2012) Viroids: how to infect a host and cause disease without encoding proteins. Biochimie 94:1474-1480

48. Navarro B, Pantaleo V, Gisel A, Moxon S, Dalmay T, Bisztray G, Di Serio F, Burgyan J (2009) Deep sequencing of viroid-derived small RNAs from grapevine provides new insights on the role of RNA silencing in plant-viroid interaction. PLoS One 4:e7686

49. Nohales MA, Flores R, Daròs JA (2012) A viroid RNA redirects host DNA ligase 1 to act as an RNA ligase. Proc Natl Acad Sci USA 109:13805-13810

50. Owens RA (2007) Potato spindle tuber viroid: the simplicity paradox resolved? Mol Plant Pathol 8:549-560

51. Owens RA, Flores R, Di Serio F, Li S-F, Pallás V, Randles JW, Sano T, Vidalakis G (2012) Viroids. In King AMQ, Adams MJ, Carstens EB, Lefkowitz EJ (eds), Virus taxonomy: ninth report of the International Committee on Taxonomy of Viruses, Elsevier/ Academic Press, London UK, pp 1221-1234

52. Rezaian MA (1990) Australian grapevine viroid-evidence for extensive recombination between viroids. Nucleic Acids Res 18:1813-1818

53. Rubino L, Di Serio F, Martelli GP (2003) Viroid-like RNAs. In: Hadidi A, Flores R, Randles JW, Semancik JS (eds) Viroids. CSIRO Publishing, Collingwood, pp 76-86

54. Sano T, Candresse T, Hammond RW, Diener TO, Owens RA (1992) Identification of multiple structural domains regulating viroid pathogenicity. Proc Natl Acad Sci USA 89:10104-10108 
55. Schmitz A, Riesner D (1998) Correlation between bending of the $\mathrm{VM}$ region and pathogenicity of different potato spindle tuber viroid strains. RNA 4:1295-1303

56. Semanicik JS, Vidalakis G (2005) The question of Citrus viroid IV as a cocadviroid. Arch. Virol 150:1059-1067

57. Szychowski JA, Vidalakis G, Semancik JS (2005) Host-directed processing of Citrus exocortis viroid. J Gen Virol 86:473-477

58. Singh RP, Dilworth AD (2009) Tomato chlorotic dwarf viroid in the ornamental plant Vinca minor and its transmission through tomato seed. Eur J Plant Pathol 123:111-116

59. Singh RP, Nie X, Singh M (1999) Tomato chlorotic dwarf viroid: an evolutionary link in the origin of viroids. J Gen Virol 80:2823-2828

60. Spieker RL (1996) In vitro-generated "inverse" chimeric Coleus blumei viroids evolve in vivo into infectious RNA replicons. J Gen Virol 77:2839-2846

61. Tamura K, Stecher G, Peterson D, Filipski A, Kumar S (2013) MEGA6: Molecular evolutionary genetics analysis version 6.0. Mol Biol Evol 30:2725-2729

62. Tsagris EM, Martinez de Alba AE, Gozmanova M, Kalanditis K (2008) Viroids. Cell Microbiol 10:2168-2179

63. Verhoeven JT, Meekes ET, Roenhorst JW, Flores R, Serra P (2013) Dahlia latent viroid: a recombinant new species of the family Pospiviroidae posing intriguing questions about its origin and classification. J Gen Virol 94:711-719

64. Verhoeven JThJ, Jansen CCC, Botermans M, Roenhorst JW (2010) Epidemiological evidence that vegetatively propagated solanaceous plant species act as sources of Potato spindle tuber viroid inoculum for tomato. Plant Pathol 59:3-12

65. Verhoeven JThJ (2010) Identification and epidemiology of pospiviroids Thesis, Wageningen University, pp 136

66. Verhoeven JThJ, Roenhorst JW, Owens RA (2011) Mexican papita viroid and Tomato planta macho viroid belong to a single species in the genus Pospiviroid. Arch Virol 156:1433-1437

67. Wu Q, Wang Y, Cao M, Pantaleo V, Burgyan J, Li W-X, Ding S-W (2012) Homology-independent discovery of replicating pathogenic circular RNAs by deep sequencing and a new computational algorithm. Proc Natl Acad Sci USA 109:3938-3943

68. Zhang Y, Singh K, Kaur R, Qiu W (2011) Association of a novel DNA virus with the grapevine vein-clearing and vine decline syndrome. Phytopathology 101:1081-1090

69. Zhong X, Archual AJ, Amin AA, Ding B (2008) A genomic map of viroid RNA motifs critical for replication and systemic trafficking. Plant Cell 20:35-47 